\title{
The Role of Fetal MRI-based Texture Analysis in Differentiating Congenital Pulmonary Airway Malformation and Pulmonary Sequestration
}

\author{
(1) Orkun Sarığlu1, (1) Fatma Ceren Sarığlu², (1) Bahar Konuralp Atakul33, (1) Deniz Öztekin4, \\ (1) Özgür Öztekin5
}

\begin{abstract}
1izmir Democracy University Faculty of Medicine, Buca Seyfi Demirsoy Training and Research Hospital, Clinic of Radiology, İzmir, Turkey 2University of Health Sciences Turkey, Tepecik Training and Research Hospital, Clinic of Radiology, Division of Pediatric Radiology, Izmir, Turkey ${ }^{3}$ Balıkesir Atatürk City Hospital, Clinic of Obstetrics and Gynecology, Division of Perinatology, Balıkesir, Turkey ${ }^{4}$ Bakırçay University Faculty of Medicine, Çiğli Training and Research Hospital, Clinic of Obstetrics and Gynecology, İzmir, Turkey ${ }^{5}$ Bakırçay University Faculty of Medicine, Çiğli Training and Research Hospital, Clinic of Radiology, İzmir, Turkey
\end{abstract}

\begin{abstract}
Aim: The purpose of our study was to evaluate the diagnostic performance of fetal magnetic resonance imaging (MRI)-based texture analysis (TA) to differentiate the two most common lung malformations, congenital pulmonary airway malformation (CPAM) and pulmonary sequestration (PS).

Materials and Methods: This retrospective single-center study included 24 patients with CPAM and 8 patients with PS who had a fetal MRI examination between January 2015 and December 2020. T2-weighted coronal images were used for TA. One reader designated the malformation borders and drew a region-of-interest for TA. The differences in values of the texture features between the groups were assessed and receiver operating characteristic curves were calculated for each statistically significant feature. P-value $<0.05$ was considered statistically significant.

Results: Forty-eight texture features were calculated for each malformation. Twenty features on T2-weighted images were significantly different between the CPAMSs and PSs. Among these, short-run high gray-level emphasis and long-run emphasis, which are gray-level runlength matrix features parameters, had the largest area under the curves: 0.956 (sensitivity 87\%, specificity 95\%) and 0.943 (sensitivity $87 \%$, specificity $85 \%$ ), respectively.
\end{abstract}

Conclusion: Our results suggest that fetal MRI-based TA may be used to distinguish CPAMs from PSs in fetuses with uncertain pulmonary findings prior to birth.

Keywords: Fetal, magnetic resonance imaging, computer-assisted image processing, lung malformation

\section{Introduction}

Congenital lung malformations (CLMs) are being noticed prior to birth more frequently nowadays by means of innovations in fetal ultrasonography (US) and magnetic resonance imaging (MRI) (1). The frequency of CLMs has been suggested to be 1 in 2,000 to 2,500 live births in studies conducted recently $(2,3)$. Despite the fact that radiologists and perinatologists are generally able to make a specific diagnosis antenatally by fetal US, similar images in different abnormalities can complicate the diagnostic process. Thus, fetal MRI first came into use as a supplementary 
examination and then became one of the routine imaging methods for the evaluation of CLMs in most centers (4-6). Nevertheless, it can still be impossible to differentiate the two most commonly encountered malformations, congenital pulmonary airway malformation (CPAM) and pulmonary sequestration (PS), by means of fetal MRI (7).

Texture analysis (TA) is a mathematical program that produces extensive numerical information from radiologic images within a region-of-interest (ROI) (8). In recent years, the role of TA in differentiating similar-looking tumors in various radiological examinations or predicting outcomes in different clinical conditions has been evaluated and has demonstrated promising results (9-12). However, there is inadequate data regarding fetal MRI-based TA in the literature. To our knowledge, to date, no prior studies have been conducted to examine the role of TA in distinguishing CLMs in the antenatal period.

Based on positive results regarding texture studies, we speculated that different malformations might be distinguished antenatally despite having similar images in fetal MRI. Hence, the purpose of our study was to assess the diagnostic performance of fetal MRI-based TA to differentiate between CPAM and PS.

\section{Materials and Methods}

\section{Patient Selection}

Ethical approval for this study was obtained from the University of Health Sciences Turkey, Tepecik Training and Research Hospital, Clinical Researches Ethics Committee (approval number: 2021/03-12, date: 24.03.2021). Informed consent was waived due to the retrospective nature of the study. The radiology and perinatology archives of a single tertiary center were scanned retrospectively to determine fetuses who were evaluated due to CLMs and had a fetal MRI examination between January 2015 and December 2020. Those patients who were diagnosed as CPAM or PS based on postnatal computed tomography (CT) findings were included in this study. Four patients with severe motion artifacts were excluded because of poor imaging quality. Finally, a total of 32 patients (24 CPAM and 8 PS) were included in this study.

\section{MRI Protocol}

Fetal MRI examinations were performed using a 1.5-T MRI system (Siemens Aera, Germany). Maternal sedation was not required in any of the scannings. While the mother was in the supine position, a torso phased array coil was placed on the pelvis, especially focusing on the fetus.
T2-weighted Half-Fourier acquisition single-shot turbospin-echo (HASTE) sequences in axial, sagittal, and coronal planes were acquired separately for the head and the body of the fetus. True fast imaging with steady-state free precession, Dixon T1-weighted, and echo-planar sequences were included in the protocol in certain conditions to obtain more information about the abnormality. Overlapping or interspacing was not preferred in the HASTE sequences of the fetal body. The HASTE sequence was acquired with the following parameters: time to repetition/time to echo, 1,100/122 ms; slice thickness, 4-5 mm; field of view (FOV), 300-400 mm; matrix, 256×256; flip angle, 180; number of excitations, 1 .

\section{MRI Interpretation and TA}

MRI images were examined by two readers (one pediatric radiologist with 4 years of experience and a 9-year experienced radiologist) in consensus. The site of the lesion (right or left) and the maximum diameter of the lesion were recorded.

TA was performed using the freely available software LIFEx (Version 5.1, www.lifexsoft.org) (13). Two radiologists who also evaluated the MRI examinations performed the TA measurements in consensus. Coronal T2-weighted HASTE sequences in Digital Imaging and Communications in Medicine format were obtained and entered into the LifeX program for the ROI delineation. The ROI drawings were performed manually by including the whole lesion on the image for which the largest lesion diameter was observed (Figure 1), as demonstrated previously in the literature $(14,15)$. The number of gray levels was adjusted to 7 bits
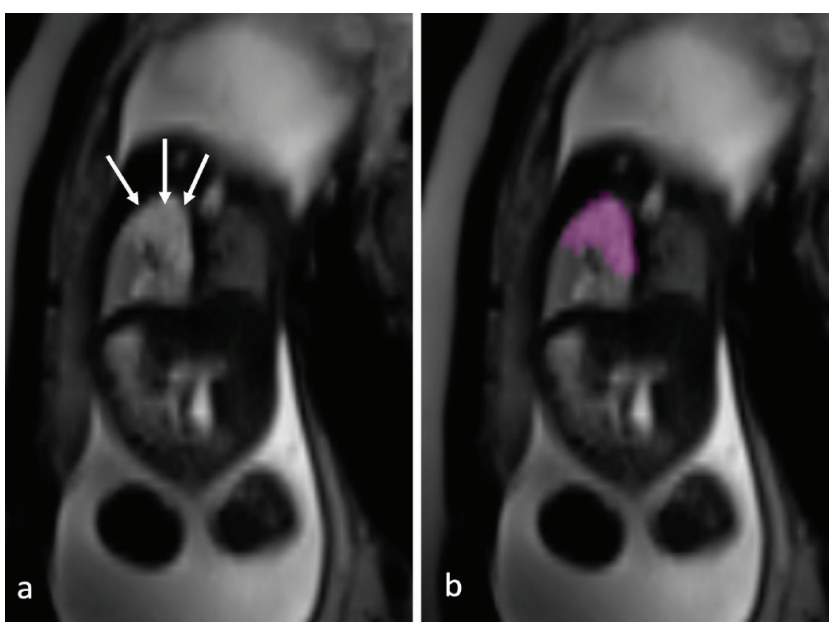

Figure 1. (a) A hyperintense lesion (arrows) is seen in the left lung in coronal T2-weighted fetal MRI. (b) ROI was drawn for the texture analysis

MRI: Magnetic resonance imaging, ROI: Region-of-interest 
(128) to ensure uniformity. An automatic measurement was performed between mean \pm 3 standard deviations (SDs) of the $\mathrm{ROI}$ content to specify intensity rescaling numbers. The minimum and maximum limits were accepted as the mean value of ROI - 3 SD and +3 SD, respectively. Numbers above or below mean $\pm 3 \mathrm{SDs}$ at the beginning were adjusted to mean \pm 3 SDs. Furthermore, the voxel widths in the $X-Y-Z$ directions were normalized as $X=0.5 \mathrm{~mm}, Y=0.5 \mathrm{~mm}$, and $Z=2.5 \mathrm{~mm}$, after counting their mean \pm 3 SDs. Finally, forty-eight TA features including six first-order (histogram) statistics, thirty-two second-order statistics, seven conventional, and three shape features were extracted for each patient.

\section{Statistical Analysis}

Statistical analysis was performed using the Statistical Package for the Social Sciences version 22.0 (SPSS Inc., Chicago, IL, USA). Continuous variables were expressed as means and SD and categorical variables as frequency counts and percentages. The distribution of the variables was examined by Kolmogorov Smirnov and Shapiro-Wilk tests. Chi-square test or Fisher's exact test was used to compare categorical variables between the two patient groups (CPAM and PS). The comparison of continuous variables was performed by the Mann-Whitney $U$ test or independent t-test. Receiver operating characteristic (ROC) curve analyses were constructed for each statistically significant feature to assess the performance of TA for making a diagnosis. P-values less than 0.05 were considered statistically significant.

\section{Results}

\section{Patient Characteristics and MRI Features}

Thirty-two patients (24 CPAM and 8 PS) were included in this study. The mean gestational age at the time of fetal MRI examination was $24.3 \pm 3.3(20-33)$ weeks. The mean maternal age was 28.2 \pm 5.2 (20-39) years. None of the demographic features showed a statistical difference between the two groups (Table I). Thirteen (40.6\%) of the malformations were located in the left hemithorax. The mean maximum diameter of the malformations was $22.91 \pm 6.4 \mathrm{~mm}$. PSs were all located in the left hemithorax. The left-sided location of the PS group was statistically significant $(p=0.007)$. In the postnatal period, CT examination was performed on all patients in order to detect a systemic feeding vessel.

\section{Texture Analysis}

Forty-eight texture features were calculated for each malformation on coronal T2-weighted HASTE images. Twenty features including 5 gray-level co-occurrence matrix (GLCM) features, 7 gray-level run-length matrix (GLRLM) features, 1 neighborhood gray-level difference matrix (NGLDM) feature, 6 gray-level zone length matrix (GLZLM) features, and 1 shape feature were significantly different between the CPAMSs and PSs (Table II).

Among the GLCM features, homogeneity, entropylog10, and entropy-log2 values were higher in the PS group while energy and dissimilarity values were found to be lower in those patients with PS. Of the GLRLM parameters, long-run emphasis (LRE), long-run high graylevel emphasis (LRHGE), gray-level non-uniformity (GLNU), and run length non-uniformity (RLNU) values were greater while short-run emphasis (SRE), short-run high gray-level emphasis (SRHGE), and run percentage values were lower in the PS group compared to the CPAMs. The NGLDM contrast feature demonstrated lower values in the PS group. In those patients with PS, long-zone emphasis (LZE) and GLNU, which are two of the GLZLM parameters, showed higher values. On the other hand, short-zone emphasis (SZE), short-zone high gray-level emphasis (SZHCE), long-zone high gray-level emphasis (LZHGE), and zone percentage (ZP) were the other parameters of GLZLM which were seen to be lower in the PS group. Lastly, one of the shape features, namely compacity, was higher in the PS group.

The ROC curve analysis results with optimal cut-off values to differentiate CPAM and PS according to TA values

Table I. Demographic data and MRI features

\begin{tabular}{|l|l|l|l|}
\hline & CPAM (n=24) & PS (n=8) & p-value \\
\hline Gestational week at fetal MRI & $23.96 \pm 3.1$ & $25.63 \pm 4.0$ & 0.117 \\
\hline Maternal age & $28.79 \pm 5.2$ & $26.75 \pm 5.3$ & 0.360 \\
\hline Birth weight (kg) & $3312 \pm 254$ & $3245 \pm 375$ & 0.265 \\
\hline Location (left/right) & $11 / 13$ & $8 / 0$ & $\mathbf{0 . 0 0 7}$ \\
\hline Maximum diameter (mm) & $23.79 \pm 6.5$ & $20.25 \pm 5.7$ & 0.191 \\
\hline CPAM: Congental &
\end{tabular}

CPAM: Congenital pulmonary airway malformation, PS: Pulmonary sequestration, MRI: Magnetic resonance imaging 


\begin{tabular}{|c|c|c|c|}
\hline Texture features & $\operatorname{CPAM}(n=24)$ & PS $(n=8)$ & p-value \\
\hline \multicolumn{4}{|l|}{ Histogram } \\
\hline $\begin{array}{l}\text { Skewness } \\
\text { Kurtosis } \\
\text { Excess kurtosis } \\
\text { Entropy-log10 } \\
\text { Entropy-log2 } \\
\text { Energy }\end{array}$ & $\begin{array}{l}-0.1984 \pm 0.3905 \\
2.9420 \pm 0.5405 \\
-0.0575 \pm 0.5404 \\
1.7808 \pm 0.0959 \\
5.9150 \pm 0.3201 \\
0.0197 \pm 0.0042\end{array}$ & $\begin{array}{l}-0.3160 \pm 0.5233 \\
2.9287 \pm 0.7976 \\
-0.0710 \pm 0.7972 \\
1.8512 \pm 0.0603 \\
6.1475 \pm 0.2047 \\
0.0167 \pm 0.0033\end{array}$ & $\begin{array}{l}0.572 \\
0.632 \\
0.632 \\
0.067^{*} \\
0.064^{*} \\
0.068\end{array}$ \\
\hline \multicolumn{4}{|l|}{ GLCM } \\
\hline $\begin{array}{l}\text { Homogeneity } \\
\text { Energy } \\
\text { Contrast } \\
\text { Correlation } \\
\text { Entropy-log10 } \\
\text { Entropy-log2 } \\
\text { Dissimilarity }\end{array}$ & $\begin{array}{l}0.1159 \pm 0.0207 \\
0.0093 \pm 0.0077 \\
651.3333 \pm 240.4242 \\
0.2230 \pm 0.2532 \\
2.2700 \pm 0.4608 \\
7.5395 \pm 1.5345 \\
20.0250 \pm 4.2173\end{array}$ & $\begin{array}{l}0.1590 \pm 0.0232 \\
0.0029 \pm 0.0029 \\
438.1250 \pm 247.5897 \\
0.4752 \pm 0.2721 \\
2.7450 \pm 0.3836 \\
9.1237 \pm 1.2829 \\
15.3900 \pm 4.5875\end{array}$ & $\begin{array}{l}<0.001 \\
\mathbf{0 . 0 1 3 ^ { * }} \\
0.051 \\
0.064 \\
\mathbf{0 . 0 1 8} \\
\mathbf{0 . 0 1 5} \\
\mathbf{0 . 0 2 9}\end{array}$ \\
\hline \multicolumn{4}{|l|}{ GLRLM } \\
\hline $\begin{array}{l}\text { SRE } \\
\text { LRE } \\
\text { LGRE } \\
\text { HGRE } \\
\text { SRLGE } \\
\text { SRHGE } \\
\text { LRLGE } \\
\text { LRHGE } \\
\text { GLNU } \\
\text { RLNU } \\
\text { RP }\end{array}$ & $\begin{array}{l}0.9907 \pm 0.0037 \\
1.0400 \pm 0.0156 \\
0.0031 \pm 0.0042 \\
4614.1666 \pm 7.1728 \\
0.0030 \pm 0.0040 \\
4572.9166 \pm 15.7367 \\
0.0034 \pm 0.0047 \\
4879.1666 \pm 428.2818 \\
5.7508 \pm 4.9567 \\
336.5291 \pm 350.7447 \\
0.9872 \pm 0.0049\end{array}$ & $\begin{array}{l}0.9821 \pm 0.0094 \\
1.1612 \pm 0.1693 \\
0.0050 \pm 0.0074 \\
4610.0000 \pm 11.9522 \\
0.0049 \pm 0.0071 \\
4521.2500 \pm 42.2365 \\
0.0061 \pm 0.0097 \\
5092.5000 \pm 359.9503 \\
18.1075 \pm 27.6785 \\
1212.6250 \pm 2044.9866 \\
0.9748 \pm 0.0135\end{array}$ & $\begin{array}{l}0.003 \\
<0.001 \\
0.862 \\
0.497 \\
0.761 \\
<0.001 \\
0.896 \\
<0.001 \\
\mathbf{0 . 0 1 3} \\
\mathbf{0 . 0 1 5} \\
\mathbf{0 . 0 0 6}\end{array}$ \\
\hline \multicolumn{4}{|l|}{ NGLDM } \\
\hline $\begin{array}{l}\text { Coarseness } \\
\text { Contrast } \\
\text { Busyness }\end{array}$ & $\begin{array}{l}0.0239 \pm 0.0126 \\
2.7074 \pm 1.7891 \\
0.0142 \pm 0.0058\end{array}$ & $\begin{array}{l}0.0148 \pm 0.0105 \\
1.2028 \pm 0.5543 \\
0.0422 \pm 0.0785\end{array}$ & $\begin{array}{l}0.107 \\
\mathbf{0 . 0 1 2 *} \\
0.459^{*}\end{array}$ \\
\hline \multicolumn{4}{|l|}{ GLZLM } \\
\hline $\begin{array}{l}\text { SZE } \\
\text { LZE } \\
\text { LGZE } \\
\text { HGZE } \\
\text { SZLGE } \\
\text { SZHGE } \\
\text { LZLGE } \\
\text { LZHGE } \\
\text { GLNU } \\
\text { ZLNU } \\
\text { ZP }\end{array}$ & $\begin{array}{l}0.8919 \pm 0.0307 \\
1.6783 \pm 0.3355 \\
0.0028 \pm 0.0036 \\
4599.5833 \pm 81.3729 \\
0.0023 \pm 0.0029 \\
4103.7500 \pm 181.7023 \\
0.0076 \pm 0.0143 \\
7732.9166 \pm 1520.55 \\
4.5292 \pm 3.6623 \\
210.8833 \pm 196.3578 \\
0.8520 \pm 0.0481\end{array}$ & $\begin{array}{l}0.8268 \pm 0.0886 \\
3.9300 \pm 3.7660 \\
0.0036 \pm 0.0045 \\
4611.2500 \pm 121.9411 \\
0.0024 \pm 0.0028 \\
3743.7500 \pm 356.5684 \\
0.0238 \pm 0.0526 \\
3738.7500 \pm 1614.46 \\
10.1137 \pm 11.9746 \\
377.1250 \pm 344.4616 \\
0.7265 \pm 0.1278\end{array}$ & $\begin{array}{l}\mathbf{0 . 0 1 1} \\
\mathbf{0 . 0 0 2} \\
0.794 \\
0.948^{*} \\
0.433 \\
\mathbf{0 . 0 0 1} \\
0.486 \\
\mathbf{0 . 0 0 2} \\
\mathbf{0 . 0 2 1} \\
0.058 \\
\mathbf{0 . 0 0 2}\end{array}$ \\
\hline \multicolumn{4}{|c|}{ Conventional indices } \\
\hline $\begin{array}{l}\text { Q1 } \\
\text { Q2 } \\
\text { Q3 } \\
\text { Min } \\
\text { Mean } \\
\text { Std } \\
\text { Max }\end{array}$ & $\begin{array}{l}396.9250 \pm 254.1990 \\
428.1416 \pm 270.5008 \\
460.1958 \pm 288.5882 \\
281.5666 \pm 181.9861 \\
427.0791 \pm 270.3255 \\
455.0972 \pm 116.3722 \\
544.8791 \pm 351.3055\end{array}$ & $\begin{array}{l}363.6250 \pm 212.7150 \\
410.5000 \pm 250.9222 \\
445.8750 \pm 274.9875 \\
258.7500 \pm 160.2121 \\
405.0000 \pm 244.3627 \\
405.2644 \pm 198.1508 \\
519.6250 \pm 316.8875\end{array}$ & $\begin{array}{l}0.896 \\
0.896 \\
0.948^{*} \\
0.896 \\
0.896 \\
0.843 \\
1.000\end{array}$ \\
\hline
\end{tabular}




\begin{tabular}{|c|c|c|c|}
\hline Texture features & CPAM $(n=24)$ & PS $(n=8)$ & p-value \\
\hline \multicolumn{4}{|l|}{ Shape features } \\
\hline $\begin{array}{l}\text { Volume (mL) } \\
\text { Volume (voxels) } \\
\text { Compacity }\end{array}$ & $\begin{array}{l}3.7567 \pm 3.8214 \\
676.1666 \pm 139.2360 \\
0.7638 \pm 0.3357\end{array}$ & $\begin{array}{l}5.4962 \pm 3.8065 \\
559.5000 \pm 233.5899 \\
1.0386 \pm 0.5099\end{array}$ & $\begin{array}{l}0.177 \\
0.744 \\
\mathbf{0 . 0 4 1}\end{array}$ \\
\hline \multicolumn{4}{|c|}{$\begin{array}{l}\text { *Independent t-test. Other p-values were calculated by using Mann-Whitney U test. Bold values indicate statistically significant. } \\
\text { CPAM: Congenital pulmonary airway malformation, PS: Pulmonary sequestration, GLCM: Gray-level co-occurrence matrix, GLRLM: Gray-level run-length matrix, SRE: } \\
\text { Short-run emphasis, LRE: Long-run emphasis, LGRE: Low gray-level run emphasis, HGRE: High gray-level run emphasis, SRLGE: Short-run low gray-level emphasis, } \\
\text { SRHGE: Short-run high gray-level emphasis, LRLGE: Long-run low gray-level emphasis, LRHGE: Long-run high gray-level emphasis, GLNU: Gray-level non-uniformity, } \\
\text { RLNU: Run length non-uniformity, RP: Run percentage, NGLDM: Neighborhood gray-level difference matrix, GLZLM: Gray-level zone length matrix, SZE: Short-zone } \\
\text { emphasis, LZE: Long-zone emphasis, LGZE: Low gray-level zone emphasis, HGZE: High gray-level zone emphasis, SZLGE: Short-zone low gray-level emphasis, SZHGE: } \\
\text { Short-zone high gray-level emphasis, LZLGE: Long-zone low gray-level emphasis, LZHGE: Long-zone high gray-level emphasis, ZLNU: Zone length non-uniformity, ZP: } \\
\text { Zone percentage, Q1: The first quartile, Q2: The second quartile, Q3: The third quartile, Min: Minimum, Std: Standard, Max: Maximum }\end{array}$} \\
\hline
\end{tabular}

Table III. The diagnostic performances of the texture analysis features with the cutoff values for pulmonary sequestration on coronal T2-weighted imaging

\begin{tabular}{|c|c|c|c|c|c|c|}
\hline Texture feature & $\begin{array}{l}\text { Area under the } \\
\text { curve }\end{array}$ & $\begin{array}{l}95 \% \text { Confidence } \\
\text { interval }\end{array}$ & $\begin{array}{l}\text { Cut-off } \\
\text { value }\end{array}$ & $\begin{array}{l}\text { Sensitivity } \\
(\%)\end{array}$ & $\begin{array}{l}\text { Specificity } \\
(\%)\end{array}$ & p-value \\
\hline GLCM_Homogeneity & 0.940 & $0.856-1.000$ & $>0.141$ & 87 & 83 & $<0.001$ \\
\hline CLCM_Entropy-log10 & 0.781 & $0.609-0.954$ & $>2.590$ & 75 & 75 & 0.019 \\
\hline GLCM_Entropy-log2 & 0.784 & $0.613-0.955$ & $>8.600$ & 75 & 75 & 0.018 \\
\hline GLRLM_LRE & 0.943 & $0.861-1.000$ & $>1.055$ & 87 & 85 & $<0.001$ \\
\hline GLRLM_LRHGE & 0.789 & $0.628-0.950$ & $>4835.0$ & 87 & 67 & 0.016 \\
\hline GLRLM_GLNU & 0.797 & $0.639-0.955$ & $>8.980$ & 87 & 67 & 0.013 \\
\hline GLRLM_RLNU & 0.792 & $0.633-0.951$ & $>244.0$ & 87 & 63 & 0.015 \\
\hline GLZLM_LZE & 0.865 & $0.709-1.000$ & $>2.065$ & 75 & 85 & 0.002 \\
\hline GLZLM_GLNU & 0.776 & $0.614-0.938$ & $>4.235$ & 87 & 75 & 0.021 \\
\hline Shape_Compacity & 0.745 & $0.515-0.974$ & $>0.839$ & 75 & 67 & 0.041 \\
\hline GLCM_Energy & 0.789 & $0.621-0.957$ & $<0.002$ & 75 & 75 & 0.016 \\
\hline GLCM_Dissimilarity & 0.760 & $0.540-0.981$ & $<18.75$ & 75 & 70 & 0.030 \\
\hline GLRLM_SRE & 0.852 & $0.686-1.000$ & $<0.986$ & 75 & 88 & 0.003 \\
\hline GLRLM_SRHGE & 0.956 & $0.887-1.000$ & $<4555.0$ & 87 & 95 & $<0.001$ \\
\hline GLRLM_RP & 0.828 & $0.626-1.000$ & $<0.980$ & 75 & 85 & 0.006 \\
\hline NGLDM_Contrast & 0.802 & $0.632-0.972$ & $<1.465$ & 75 & 67 & 0.012 \\
\hline GLZLM_SZE & 0.805 & $0.562-1.000$ & $<0.859$ & 75 & 85 & 0.011 \\
\hline GLZLM_SZHGE & 0.880 & $0.745-1.000$ & $<3975.0$ & 75 & 80 & 0.001 \\
\hline GLZLM_LZHGE & 0.530 & $0.576-0.946$ & $<4525.0$ & 50 & 65 & 0.002 \\
\hline GLZLM_ZP & 0.865 & $0.704-1.000$ & $<0.814$ & 75 & 80 & 0.002 \\
\hline
\end{tabular}

are displayed in Table III. Two GLRLM parameters, SRHGE and $L R E$, showed the largest area under the curve results: 0.956 (95\% confidence interval $(\mathrm{Cl}), 0.887-1)$ and 0.943 [95\% Cl 0.861-1], respectively (Figure 2). A GLRLM_SRHGE value of $<4555.0$ had the finest diagnostic results with a sensitivity of $87 \%$ and a specificity of $95 \%$ for diagnosing PS. The optimal cut-off TA value for the GLRLM_LRE was >1.055, with a sensitivity of $87 \%$ and a specificity of $85 \%$. 

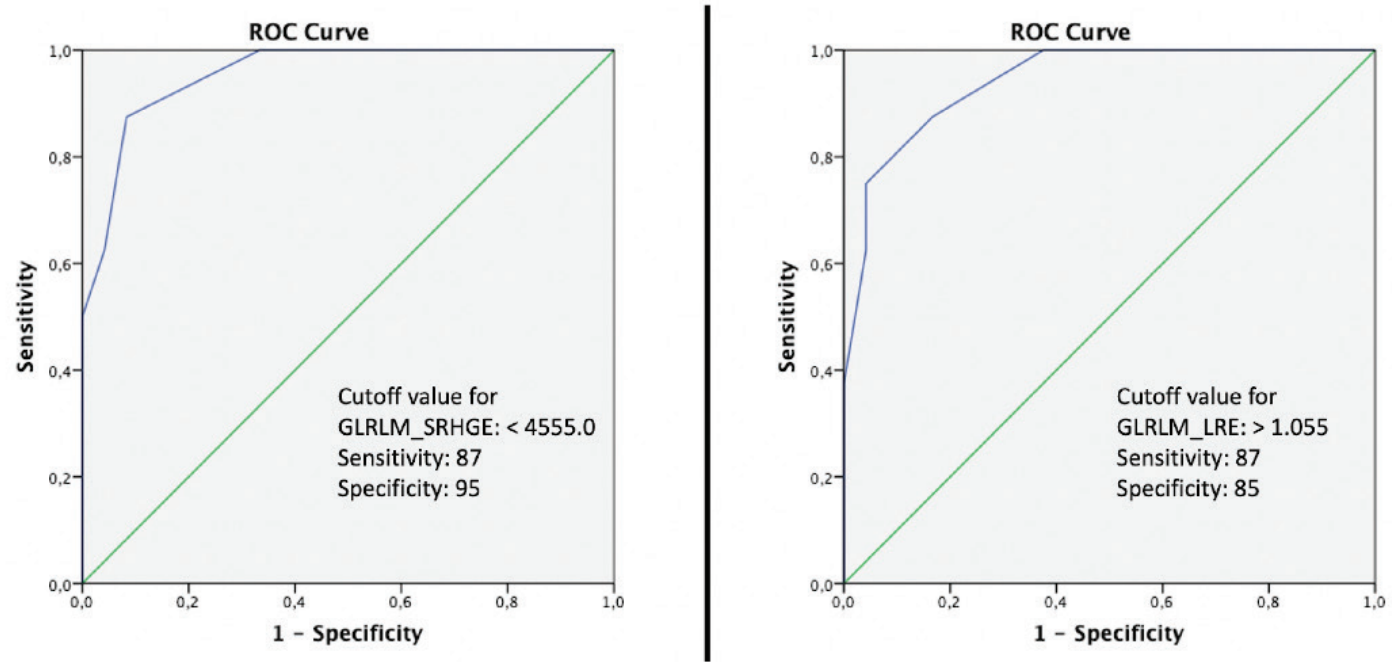

Figure 2. Receiver operating characteristics curves of the GLRLM_SRHGE and the GLRLM_LRE for differentiating pulmonary sequestration ROC: Receiver operating characteristic, GLRLM: Gray-level run-length matrix, SRHGE: Short-run high gray-level emphasis, LRE: Long-run emphasis

\section{Discussion}

In this study, we assessed the performance of TA values to differentiate between fetuses with CPAM and PS on fetal MRI examinations. As far as we know, this study is the first in the literature to analyze the texture parameters between CLMs. Our study showed that fetal MRI-based TA may help to distinguish between CPAM and PS antenatally. Several TA parameters demonstrated a significant difference between those patients with CPAM and those with PS, in addition to the favorable area under the curve results from 0.745 to 0.956 in our study. A GLRLM SRHGE value of $<4555.0$ and a GLRLM_LRE value of $>1.055$ were found to be the most significant parameters to make an accurate diagnosis with a sensitivity of $87 \%$ in both and a specificity of $95 \%$ and $85 \%$, respectively.

TA allows for the extraction of quantitative features from the medical images by means of dedicated software (16). The principle of TA is based on the knowledge that pixels in an image contain quantitative information, which may reveal the underlying pathophysiology of a texture (17). TA features are divided into subgroups as firstorder features (histogram), second-order features, shape features, and conventional indices. First-order features, also defined as histogram analysis, contain the distribution of voxel values inside an ROI (18). Second-order features describe the statistical associations between pixels (18). The GLCM shows pixel pairs in a figure towards a particular route while the GLRLM calculates the size of pixels (17). The NGLDM reflects the difference in gray-level values between a particular pixel and its neighbors (19). The
GLZLM calculates the size of uniform pixels for each grey level (15). The shape features represent the geometric factors like volume, sphericity, and compacity within an ROI.

The data obtained from TA and their meanings do not reflect any visual finding on the radiological images. In this study, we did not find any differences in the first-order and conventional features and this may indicate that CPAM and PS have a comparable distribution of gray-level intensities in fetal MRI images. The shape features also did not show any significance except for compacity, which was one of the least significant features among all significant variables. However, a number of second-order features demonstrated statistical significance in our study. To summarize the parameters of interest; SRE, SRHGE, SZE, SZHGE, LZHGE, and $Z P$ values were lower in the PS group while LRE, LRHGE, and LZE values were higher. According to these findings, we can interpret that high gray-levels are less frequent in PSs. This may be explained by the knowledge that CPAMs have cystic components (7) and this microstructural texture results in higher gray-levels in the T2-weighted MRI image. The GLRLM_SRHGE and the GLRLM_LRE values showed the highest significance in this study, supporting our theory.

The opportunity of evaluating relations between quantitative data and imaging features has brought a new point of view to various clinical conditions. However, to the best of our knowledge, TA has not been evaluated in fetal MRI images. Therefore, we did not have the chance to compare our results with the literature. Making an accurate and trustworthy diagnosis is very important in CLMs as it 
may change the management, for instance, resection or observation in asymptomatic patients $(20,21)$. Although fetal US and MRI can determine an accurate diagnosis mostly, it has also been reported that both US and MRI were unable to diagnose CLMs in approximately $25 \%$ of cases (22). It was also indicated that although fetal MRI was superior to US for correctly determining the PS, the sensitivity rate was $70 \%$. For these reasons, we evaluated the performance of TA in addition to fetal MRI, to figure out if it might help to determine the right diagnosis in the two most common CLMs, namely, CPAM and PS. Our diagnostic performance rates with fetal MRI-based TA were found to be higher than the study conducted by Mon et al. (22), in which they demonstrated the diagnostic accuracy of fetal US and MRI in CLMs.

\section{Study Limitations}

There were some limitations in this study. The retrospective nature of the study and the small number of subjects were the major limitations. Secondly, TA was performed on two-dimensional slices instead of volumetric measurement. Although three-dimensional analysis represents more extensive information, two-dimensional analysis has also been proven to provide adequate results (23). Thirdly, it is a known fact that TA values may be affected by different imaging protocols. Even though all fetal MRI examinations were performed by one scanner and had the same acquisition parameters, the FOV varied between 300-400 mm depending on the size of the fetuses. We normalized the voxel sizes and standardized the pixel discrimination to reduce this limitation (24). Finally, although TA values were measured automatically, two observers manually delineated the margins and drew the ROIs.

\section{Conclusion}

Fetal MRI-based TA provided comprehensive information regarding the two most common CLMs. Various texture parameters, particularly the GLRLM features, revealed important data to differentiate between CPAM and PS with high rates of sensitivity and specificity. By means of further trials with a larger population, TA might be used as a valuable modality to predict diagnosis in patients with CLMs prior to birth.

\section{Ethics}

Ethics Committee Approval: Ethical approval for this study was obtained from the University of Health Sciences Turkey, Tepecik Training and Research Hospital, Clinical
Researches Ethics Committee (approval number: 2021/0312, date: 24.03.2021).

Informed Consent: Informed consent was waived due to the retrospective nature of the study.

Peer-review: Externally and internally peer-reviewed.

\section{Authorship Contributions}

Concept: D.Ö., Ö.Ö., Design: F.C.S., Data Collection or Processing: O.S., F.C.S, B.K.A., Analysis or Interpretation: F.C.S., O.S., Literature Search: O.S., B.K.A., Ö.Ö., Writing: O.S.

Conflict of Interest: The authors declared no conflict of interest.

Financial Disclosure: The authors declared that this study received no financial support.

\section{References}

1. Goldstein RB. A practical approach to fetal chest masses. Ultrasound Q 2006; 22:177-94

2. Burge $D$, Wheeler R. Increasing incidence of detection of congenital lung lesions. Pediatr Pulmonol 2010; 45:104.

3. Lima JS, Camargos PA, Aguiar RA, Campos AS, Aguiar MJ. Pre and perinatal aspects of congenital cystic adenomatoid malformation of the lung. I Matern Fetal Neonatal Med 2014; 27:228-32.

4. Levine D, Barnewolt CE, Mehta TS, Trop I, Estroff J, Wong G. Fetal thoracic abnormalities: MR imaging. Radiology 2003; 228:379-88

5. Liu YP, Chen CP, Shih SL, Chen YF, Yang FS, Chen SC. Fetal cystic lung lesions: evaluation with magnetic resonance imaging. Pediatr Pulmonol 2010; 45:592-600.

6. Santos XM, Papanna R, Johnson A, et al. The use of combined ultrasound and magnetic resonance imaging in the detection of fetal anomalies. Prenat Diagn 2010; 30:402-7.

7. Biyyam DR, Chapman T, Ferguson MR, Deutsch G, Dighe MK. Congenital lung abnormalities: embryologic features, prenatal diagnosis, and postnatal radiologic-pathologic correlation. Radiographics 2010; 30:1721-38.

8. Kassner A, Thornhill RE. Texture analysis: a review of neurologic MR imaging applications. AJNR Am / Neuroradiol 2010; 31:80916

9. Sarioglu O, Sarioglu FC, Akdogan Al, et al. MRI-based texture analysis to differentiate the most common parotid tumours. Clin Radiol 2020; 75:877.

10. Sarioglu FC, Sarioglu O, Guleryuz H, Ozer E, Ince D, Olgun $\mathrm{HN}$. MRI-based texture analysis for differentiating pediatric craniofacial rhabdomyosarcoma from infantile hemangioma. Eur Radiol 2020; 30:5227-36.

11. Sandrasegaran K, Lin Y, Asare-Sawiri M, Taiyini T, Tann M. CT texture analysis of pancreatic cancer. Eur Radiol 2019; 29:106773.

12. Sarioglu O, Sarioglu FC, Capar AE, Sokmez DFB, Topkaya P, Belet $U$. The role of $C T$ texture analysis in predicting the 
clinical outcomes of acute ischemic stroke patients undergoing mechanical thrombectomy. Eur Radiol 2021; 31:6105-15.

13. Nioche C, Orlhac F, Boughdad S, et al. LIFEx: A Freeware for Radiomic Feature Calculation in Multimodality Imaging to Accelerate Advances in the Characterization of Tumor Heterogeneity. Cancer Res 2018; 78:4786-9.

14. Cannella R, Rangaswamy B, Minervini MI, Borhani AA, Tsung A, Furlan A. Value of Texture Analysis on Gadoxetic AcidEnhanced MRI for Differentiating Hepatocellular Adenoma From Focal Nodular Hyperplasia. AJR Am J Roentgenol 2019; 212:538-46.

15. Li Z, Mao Y, Huang W, et al. Texture-based classification of different single liver lesion based on SPAIR T2W MRI images. BMC Med Imaging 2017; 17:42.

16. Gillies RJ, Kinahan PE, Hricak H. Radiomics: Images Are More than Pictures, They Are Data. Radiology 2016; 278:563-77.

17. Rizzo S, Botta F, Raimondi S, et al. Radiomics: the facts and the challenges of image analysis. Eur Radiol Exp 2018; 2:36.

18. Koçak B, Durmaz EŞ, Ateş E, Kılıçkesmez Ö. Radiomics with artificial intelligence: a practical guide for beginners. Diagn Interv Radiol 2019; 25:485-95.
19. Stoecker WV, Chiang CS, Moss RH. Texture in skin images: comparison of three methods to determine smoothness. Comput Med Imaging Graph 1992; 16:179-90.

20. Aziz D, Langer JC, Tuuha SE, Ryan G, Ein SH, Kim PC. Perinatally diagnosed asymptomatic congenital cystic adenomatoid malformation: to resect or not? J Pediatr Surg 2004; 39:329-34.

21. Ng C, Stanwell I, Burge DM, Stanton MP. Conservative management of antenatally diagnosed cystic lung malformations. Arch Dis Child 2014; 99:432-7.

22. Mon RA, Johnson KN, Ladino-Torres M, et al. Diagnostic accuracy of imaging studies in congenital lung malformations. Arch Dis Child Fetal Neonatal Ed 2019; 104:372-7.

23. Ng F, Kozarski R, Ganeshan B, Goh V. Assessment of tumor heterogeneity by $\mathrm{CT}$ texture analysis: can the largest crosssectional area be used as an alternative to whole tumor analysis? Eur J Radiol 2013; 82:342-8.

24. Collewet G, Strzelecki M, Mariette F. Influence of MRI acquisition protocols and image intensity normalization methods on texture classification. Magn Reson Imaging 2004; 22:81-91. 